\title{
APOLIPOPROTEIN E genotype status affects habitual human blood mononuclear cell gene expression and its response to fish oil intervention
}

Juri C Matualatupauw ${ }^{1,2}$, Marijana Radonjic ${ }^{2,3}$, Ondine van de Rest ${ }^{1}$, Lisette CPGM de Groot $^{1}$, Johanna M Geleijnse ${ }^{1}$, Michael Müller ${ }^{1,4}$, Lydia A Afman $^{1}$

${ }^{1}$ Division of Human Nutrition, Wageningen University, 6700EV Wageningen, the Netherlands

${ }^{2}$ Microbiology and Systems Biology, TNO, 3700 AJ Zeist, The Netherlands

${ }^{3}$ Current address: EdgeLeap B.V, Utrecht, The Netherlands

${ }^{4}$ Current address: Norwich Medical School, University of East Anglia, Norwich, UK

Corresponding author: LA Afman, Division of Human Nutrition, Wageningen University, PO Box 8129, 6700 EV, Wageningen, the Netherlands. E-mail: 1ydia.afman@wur.nl. Phone: 00 31317485789.

Abbreviations: APOE, Apolipoprotein E; CRP, C-reactive protein; CVD, cardiovascular disease; DHA, docosahexaenoic acid; EPA, eicosapentaenoic acid; GSEA, gene set enrichment analysis; HOSF, high-oleic sunflower oil; IFN, Interferon; PBMC, peripheral blood mononuclear cell; qPCR, quantitative real-time polymerase chain reaction; SNP, single nucleotide polymorphism

Keywords: $A P O E 4$, fish-oil supplementation, gene-diet interactions, microarray, nutrigenomics 


\begin{abstract}
Scope

People who carry the apolipoprotein E4 (APOE4) SNP have an increased risk of cardiovascular disease (CVD). Fish-oil supplementation may help in the prevention of CVD, though inter-individual differences in the response to n-3 PUFAs have been observed. We aimed to assess the impact of $A P O E$ genotype on peripheral blood mononuclear cell (PBMC) whole genome gene expression at baseline and following a fish-oil intervention.
\end{abstract}

Methods and results

Participants received 6 months of fish-oil supplementation containing $1800 \mathrm{mg}$ of eicosapentaenoic acid and docosahexaenoic acid per day. APOE genotype and PBMC whole genome gene expression before and after supplementation were measured. We characterized the differences in gene expression profiles in carriers of APOE4 $(N=8)$ compared to noncarriers $(\mathrm{N}=15)$. At baseline, 1320 genes were differentially expressed and the fish-oil supplementation differentially regulated 866 genes between APOE4 carriers and non-carriers. Gene set enrichment analysis showed that carriers had a higher gene expression of cholesterol biosynthesis and interferon (IFN) signaling pathways. Fish-oil supplementation reduced expression of IFN-related genes in carriers only.

\title{
Conclusion
}

The increased expression of IFN signaling and cholesterol biosynthesis pathways might explain part of the association between APOE4 and CVD. Fish-oil supplementation may particularly benefit $A P O E 4$ carriers by decreasing expression of IFN-related genes. 


\section{Introduction}

Apolipoprotein $\mathrm{E}(A P O E)$ is part of several types of lipoproteins including VLDL, HDL and chylomicrons [1]. Three major isoforms exist: $A P O E 2, A P O E 3$ and $A P O E 4$, which have allele frequencies of $6 \%, 15 \%$ and $78 \%$ respectively [2]. Large meta-analyses have shown that carriers of $A P O E 4$ have a modestly increased risk of coronary heart disease $[3,4]$ and stroke [5] compared with individuals with the APOE3/APOE3 genotype, though the magnitude of this finding differs between studies and it is not always significant [4]. Furthermore APOE4 is associated with increased levels of LDL cholesterol [4-6] and triglycerides [7] in the plasma. For prevention of CVD, the European Society of Cardiology recommends two fatty fishcontaining meals a week [8]. A meta-analysis of randomized controlled trials showed that supplementary n-3 polyunsaturated fatty acids (PUFAs) decreases the risk of CVD [9]. However, this positive impact on CVD is not reported consistently; several studies find no association between n-3 PUFA supplementation and CVD risk [10]. Furthermore, interindividual differences in the response to n-3 PUFAs have been observed. This inter-individual variability may, in part, be caused by genetic factors [11].

Several studies have shown that $A P O E$ genotype influences the plasma lipid response to fishoil supplementation. Minihane et al. 2000 [12] found a significant increase in total cholesterol and a trend toward a reduction in HDL-Cholesterol in APOE4 carriers relative to APOE3/APOE3 subjects after fish-oil supplementation. In line with this, Olano-martin et al. 2010 [13] found increased total cholesterol concentrations in APOE4 carriers in response to 4 weeks docosahexaenoic acid (DHA) supplementation. Caslake et al 2008 [14] found the greatest decrease in plasma triglyceride concentration in APOE4 men after fish-oil supplementation.

We previously showed that 6 months fish-oil supplementation decreased gene expression of genes involved in inflammatory and atherogenic pathways in peripheral blood mononuclear 
cells (PBMCs) [15]. PBMCs are a subpopulation of circulating immune cells mainly consisting of monocytes and lymphocytes. These circulating immune cells are important players in the pathogenesis of CVD, especially atherosclerosis, and, therefore, are a suitable target for studying inflammatory and CVD mechanisms [16]. APOE genotype has been shown to affect immune cell response [17-19]. For example, mice monocytes/macrophages showed an increased inflammatory response when transfected to produce human APOE4 compared to $A P O E 3$ [17]. Moreover, signaling via the $A P O E$ receptors promotes conversion of macrophages from the pro-inflammatory M1 to the anti-inflammatory M2 phenotype [18], which may be decreased in $A P O E 4$ carriers because of the lower $A P O E$ concentrations that are observed with APOE4 [19]. From these results, it is thought that the altered inflammatory response of $A P O E 4$-expressing monocytes/macrophages may contribute to the higher CVD risk observed in APOE4 carriers.

By measuring PBMC whole genome gene expression with microarrays and combining this with $A P O E$ genotyping, we are able to unbiasedly study genotype-specific phenotype effects. In this study, we aimed to gain more insight in the pathways affected by APOE4 and the effect of fish-oil supplementation on these pathways by studying gene-diet interactions between APOE4 and fish-oil supplementation on whole genome gene expression. To do this, we conducted a secondary retrospective analysis according to APOE4 carrier status of the above-mentioned study [15, 20]. Firstly, we studied the effect of APOE4 on whole genome PBMC gene expression at baseline and, secondly, we studied how APOE4 influences the effects of 6 months of fish-oil supplementation on gene expression.

\section{Material and methods}

\section{Study design}


This study is a secondary retrospective analysis of a randomized, double-blind, placebocontrolled trial conducted by van de Rest et al. (2008) [20] that was originally designed to examine the effects of 6 months fish-oil supplementation on cognitive performance in 302 participants aged $\geq 65$ years. In this secondary analysis, we examine differences in whole genome gene expression between APOE4 carriers and APOE4 non-carriers before and following the fish-oil intervention. Microarray data was available in 23 subjects from the high dose fish-oil group [15], with RNA available in 92 participants. To validate findings of the microarray analyses, we performed targeted QPCR measurements in these 92 participants Design and methods of the original study were described in detail previously [20]. Briefly, participants were randomly allocated to receive a daily dose of fish-oil containing either 1800 mg or 400 mg eicosapentaenoic acid (EPA) and DHA, or a placebo oil (high-oleic sunflower oil (HOSF)) for 26 weeks. The high daily dose of fish oil provided $1093 \pm 17 \mathrm{mg}$ EPA and $847 \pm 23 \mathrm{mg}$ DHA, and the low daily dose provided $226 \pm 3 \mathrm{mg}$ EPA and $176 \pm 4 \mathrm{mg}$ DHA. The oils were administered in 6 soft gelatin capsules daily, each of which contained $900 \mathrm{mg}$ oil and $2.7 \mathrm{mg}$ tocopherol as antioxidant (Banner Pharmacaps Europe BV, Tilburg, Netherlands).

Participants were recruited according to the following exclusion criteria: current or recent $(<4$ weeks) use of fish-oil supplements or intake of fish $>4$ times/week or $>800 \mathrm{mg}$ fish-oil/d from fish as estimated by using a fish-consumption questionnaire, serious liver disease, consumption of $>4$ glasses of alcohol-containing beverages per day, unable to participate as judged by the responsible medical physician, allergy to fish or fish-oil, swallowing problems, or participation in another clinical trial $<2$ mo before the start of the trial or at the same time. Cognitive exclusion criteria were also used and were described previously [20]. Additionally, compliance with capsule use during a 2 -week placebo run-in period had to be $\geq 80 \%$ on the basis of self-report. All participants gave written informed consent to participate in the study 
and the study protocol was approved by the Medical Ethical Committee of Wageningen University, Wageningen, the Netherlands. The study was registered at clinicaltrials.gov as NCT00124852.

\section{Blood sampling and PBMC isolation}

Fasting venous blood samples were collected at baseline and after 26 weeks of intervention. Plasma free fatty acids and triglycerides were measured by gas-liquid chromatography, and C-reactive protein (CRP) concentrations were determined from measurements of highsensitivity CRP (hsCRP). For PBMC isolation, $4 \mathrm{~mL}$ blood was collected into Becton Dickinson Vacutainer Cell Preparation Tubes with sodium citrate. PBMCs were isolated immediately after blood collection according to the manufacturer's instructions. For $A P O E$ genotyping, a second blood sample was collected into a 4.5-mL EDTA Vacutainer and stored at $-80^{\circ} \mathrm{C}$.

\section{$A P O E$ genotyping}

$A P O E$ genotyping was done by the PCR-based restriction fragment length polymorphism method and restriction enzyme digestion with HhaI [21]. We retrospectively determined $A P O E$ genotype in 301 of the participants of the original study.

\section{RNA extraction and microarray}

RNA extraction and microarray methods were previously described by Bouwens et al. [15] and data can be found in Gene Expression Omnibus under accession number GSE12375. We reanalyzed the data using the current gene definitions (NuGOHs1a520180_Hs_ENTREZG MBNI custom CDF version 19.0.0) and grouped the samples based on the APOE genotyping results (Supporting Information table S1).

Microarray analysis was performed on baseline samples and on samples after 26 weeks of intervention, using human whole-genome NuGO GeneChip arrays designed by the European Nutrigenomics Organization and manufactured by Affymetrix (Affymetrix Inc, Santa Clara, 
CA). Microarrays were analyzed using MADMAX (Management and Analysis Database for Multiplatform Microarray Experiments) [22]. Expression values were normalized using the RMA (robust multichip average method) [23]. Genes with normalized expression values $>20$ on at least 5 arrays were defined as expressed and selected for further analysis. Expression values were $\log 2$-transformed. LIMMA [24] was used to calculate P-values and false discovery rate (FDR) q-values for each gene using t-tests with Bayesian correction. At baseline, genes were defined as differentially expressed between APOE4 carriers and noncarriers if they had P-values $<0.05$. The differences in response to fish-oil supplementation were calculated using the individual log ratios. Differences in genes expression changes between $A P O E 4$ carriers and non-carriers were defined as significantly different if they had Pvalues $<0.05$.

Pathway analysis and upstream transcription regulators analyses were performed using QIAGEN's Ingenuity Pathway Analysis (IPA, QIAGEN Redwood City, www.qiagen.com/ingenuity). We also performed pathway analysis using gene set enrichment analysis (GSEA; http://www.broad.mit.edu/gsea) [25]. Briefly, genes were ranked based on the t-statistic and analyzed for over- or underrepresentation in predefined gene sets. Gene sets were derived from Biocarta, KEGG, Reactome and Wikipathways pathway databases.

Genesets with a false discovery rate $<0.1$ were considered significantly enriched

\section{(Supporting Information tables S2-S5).}

\section{QPCR}

For qPCR, RNA was available of PBMCs of 92 participants of which 31 were carriers and 61 were non-carriers of $A P O E 4$. RNA was reverse transcribed using a cDNA synthesis kit (RevertAid First Strand cDNA Synthesis kit, Thermo Scientific, Leusden, the Netherlands) and analyzed by qPCR (SensiMix SYBR No-ROX, Bioline, London, UK) on a CFX384 RealTime System (C1000 Thermal Cycler, Biorad, Veenendaal, The Netherlands). qPCR data 
were normalized using the RPLP0 housekeeping gene. Genes for qPCR were selected from the differentially expressed pathways between APOE4 carriers and non-carriers. Genes needed to be differently expressed between APOE4 carriers and non-carriers and have an expression value above 200. Primers sequences are shown in Table 1.

\section{Statistics}

Statistical analysis of subject characteristics and qPCR findings was performed using IBM SPSS Statistics, Version 22.0.0.1. A two-sided $\mathrm{P}<0.05$ was considered significant. Subject characteristics were reported as mean \pm sd. Baseline characteristics of APOE4 carrier and non-carrier groups were compared using independent t-tests. QPCR results were analyzed using independent t-tests.

Power calculations were performed retrospectively for the HMGCS1 gene. Power was calculated to be $83 \%$, using the mean expression values and standard deviations as observed (218.7 and 184.8 for APOE4 carriers; and 28.2 and 23.5 for APOE4 non-carriers). 


\section{Results}

\section{Participant characteristics}

The original study consisted of 302 participants. Here, we focused on a subgroup of participants who received $1800 \mathrm{mg}$ fish-oil and on whom PBMC whole genome gene expression microarray analysis was performed $(\mathrm{N}=23)$. APOE genotyping showed that 8 of the 23 participants were carriers of APOE4. Baseline characteristics of carriers and noncarriers are presented in Table $\mathbf{2}$. We found no differences in any parameters between the groups. Participants received daily fish-oil supplementation containing $1800 \mathrm{mg}$ of EPA and docosahexaenoic acid (DHA) for 6 months. We found no difference in the changes in any parameters between APOE4 carriers and non-carriers (Table 2).

\section{Baseline}

To determine the effects of $A P O E 4$ on gene expression, baseline expression profiles were compared between APOE4 carriers and non-carriers. Of the 13027 genes found to be expressed, 1320 genes were differentially expressed between carriers and non-carriers of the APOE4 SNP at $\mathrm{P}<0.05$ (Figure 1). No genes showed an FDR $\mathrm{q}<0.05$.

To gain further insight into the biological processes affected by APOE4, we performed pathway analysis using Ingenuity Pathway Analysis on the set of differentially expressed genes at baseline. Figure 2 shows the top 10 pathways that were enriched in the comparison of $A P O E 4$ carriers and non-carriers. Several of these pathways were related to either cholesterol biosynthesis or interferon (IFN) signaling. Furthermore, GSEA showed several gene sets related to cholesterol biosynthesis or IFN signaling as upregulated. (Supporting

\section{Information table S2).}

Further inspection of the function of the genes related to cholesterol biosynthesis, showed that many of these genes are encoding enzymes involved in de novo synthesis of cholesterol. These genes were found to be more highly expressed in APOE4 carriers than in non-carriers. 
Genes involved in cholesterol uptake (LDLR and CD36) and efflux (ABCA1, ABCG1 and SCARB1) showed no difference in expression in APOE4 carriers compared to non-carriers. We observed a higher expression of genes involved in IFN signaling in carriers compared to non-carriers of $A P O E 4$. These genes included IFN (alpha, beta and omega) receptor 1 (IFNAR1) as well as the intracellular signaling molecules Signal Transducers and Activator of Transcription (STAT)1 and STAT2. Besides IFN signaling genes, we also found a higher expression of many IFN target genes in carriers of the APOE4 SNP compared to non-carriers (Table 3 and Figure 3).

To examine potential regulators of the observed gene expression differences between carriers and non-carriers, we performed Ingenuity upstream regulator analysis. IFNL1, IFNA2 and IFNG were identified as potential transcriptional regulators of the observed gene expression differences. These regulators and their targets are shown in Figure 3. Additionally, several other molecules that are involved in IFN signaling were identified as potential upstream regulators (Table 4).

\section{Fish-oil intervention effect}

To examine the effect of APOE4 on the gene expression response to fish-oil supplementation, we determined the genes that were differentially regulated in response to fish-oil supplementation in carriers compared to non-carriers of APOE4. We found that 866 genes showed a different change in expression caused by fish-oil supplementation between carriers and non-carriers at $\mathrm{P}<0.05$ (Figure 1). No genes showed an FDR $\mathrm{q}<0.05$.

Figure 4 shows the enriched pathways when comparing the response to fish-oil supplementation between carriers and non-carriers of APOE4. The top 2 pathways are both related to IFN signaling. Furthermore, GSEA showed several gene sets related to IFN signaling as downregulated when comparing the response to fish-oil supplementation between carriers and non-carriers of APOE4 (Supporting Information table S5). 
In carriers of the $A P O E 4$ allele, genes in these IFN signaling pathways were found to be mostly downregulated, whereas non-carriers mostly showed an upregulation in response to n3 PUFA supplementation when comparing gene expression after supplementation to expression at baseline.

To examine potential regulators of the differentially expressed genes, we performed Ingenuity upstream regulator analysis. This revealed many IFN-related genes as potential upstream transcriptional regulators, including IFNL1, IFNA2 and IFNG (Table 5). The genes regulated by these cytokines were found to be largely downregulated in APOE4 carriers and largely upregulated in non-carriers, as shown in Figure 5.

Comparison of gene expression profiles after 6 months fish-oil supplementation between carriers and non-carriers of $A P O E 4$ showed that both cholesterol biosynthesis as well as IFN signaling pathways were no longer enriched in the comparison between carriers and noncarriers of APOE4 (data not shown).

\section{QPCR validation of the microarray findings}

QPCR was used to determine the expression of five genes selected from the cholesterol biosynthesis and IFN signaling pathways in all available RNA samples. We determined gene expression of HMGCR, HMGCS1, IFITM1, STAT1 and TAP1 in a total of 92 participants, of which 31 were APOE4 carriers and 61 were APOE4 non-carriers. We found that expression of HMGCS1 and STAT1 was significantly higher in APOE4 carriers compared to non-carriers at baseline (Figure 6). Mean expression of HMGCR, IFITM1 and TAP1 was higher in APOE4 carriers compared to non-carriers, though these differences were not significant. 


\section{Discussion}

This study examined the effect of APOE4 on whole genome gene expression in PBMCs at baseline and in response to fish-oil supplementation. Expression of genes involved in cholesterol biosynthesis as well as IFN signaling and IFN target genes was found to be higher in APOE4 carriers compared to non-carriers. Interestingly, 6 months of fish-oil supplementation decreased IFN-related gene expression in APOE4 carriers.

The increased expression of genes in the IFN signaling pathway and IFN-regulated genes in PBMCs of APOE4 carriers at baseline may point towards a systemic pro-inflammatory state. Consistent with our findings, APOE4 has been linked to increased systemic inflammation as carriers of the APOE4 allele have been shown to have higher plasma levels of the proinflammatory cytokines IL-8 and TNF- $\alpha$ [26], and lower levels of the anti-inflammatory cytokine IL-10 [27]. Furthermore, IFNG produced by T-lymphocytes is highly expressed in atherosclerotic lesions and affects many processes involved in the pathogenesis of atherosclerosis [28]. Increased expression of genes in the IFN signaling pathway as well as increased expression of IFN targets in PBMCs of APOE4 carriers may unfavorably prime these cells and may increase the risk of atherosclerosis. The question remains whether the observed increase in IFN-related gene expression is caused by APOE4 directly, or whether it is a consequence of indirect factors that are affected by APOE4.

Literature on the role of fish-oil or n-3 PUFAs on IFN pathways and IFN-regulated genes is scarce. It is possible that previous studies that did not take APOE genotype into account found no effects, as we show in the current study that IFN signaling-related gene expression is either upregulated or downregulated dependent on $A P O E$ genotype. We identified one mouse study, in which a high intake of n-3 PUFAs was shown to lead to diminished STAT1 phosphorylation after ex-vivo stimulation of immune cells with IFN-gamma [29]. A similar mechanism could be present in our APOE4 carriers, though, to our knowledge, no studies 
exist that have examined the role of $A P O E$ genotype in this context. One relatively small study in 35 Alzheimer's disease patients examined the interaction between APOE genotype and 6 months of fish-oil supplementation on several inflammatory cytokines in the plasma, but found no effects of $A P O E$ genotype [30].

In contrast to $A P O E 4$ carriers, non-carriers showed an upregulation of IFN pathways. Previously, we examined the effects of fish-oil supplementation in this population and found anti-inflammatory and anti-atherogenic gene expression changes when not taking $A P O E$ genotype into account [15]. These changes were not found in genes related to IFN signaling pathways. This indicates that fish-oil supplementation may have pro-inflammatory as well as anti-inflammatory effects in APOE4 non-carriers. Further research is required to fully understand the $A P O E$ genotype-specific effects of fish-oil supplementation on inflammatory signaling.

APOE4 is associated with an increased risk of CVD [4]. Partly, this is thought to be mediated by increased LDL-cholesterol and triglyceride plasma concentrations in APOE4 carriers [31]. In the full study group of 301 participants, we also observed significantly higher LDLcholesterol levels in the plasma of $A P O E 4$ carriers compared to non-carriers. Our microarray data point towards an increase in cholesterol synthesis as reflected by a higher expression of several genes encoding enzymes involved in the synthesis of cholesterol, including HMGCoA reductase (HMGCR), the enzyme catalyzing the rate-limiting step in the synthesis of cholesterol [32]. Expression of this gene and other genes involved in cholesterol biosynthesis is known to be controlled by the SREBP-2 transcription factor through a negative feedback system [33]. Therefore, the higher expression of cholesterol biosynthesis genes in APOE4 carriers might suggests a lower intracellular cholesterol concentration and, consequently, a reduced activity of SREBP-2. However, the SREBP-2 gene itself was not differentially expressed between APOE4 carriers and non-carriers and, based on the increased LDL- 
cholesterol concentrations in APOE4 carriers that we observed in the full study group of 301 participants and is consistently reported in literature, one might expect increased intracellular cholesterol levels. Whether cholesterol levels are actually increased in the cell requires further investigation.

The role of increased cholesterol synthesis in monocytes remains unclear. It may promote cholesterol accumulation in these cells, which is an important first step in foam cell formation and may, therefore, potentially be related to the increased CVD risk in APOE4 carriers. Additionally, in a study by Gerdes et al. (2000) [34], treatment with simvastatin, an inhibitor of the HMGCR protein, abolishes the APOE4-related 2-fold increase in mortality after a previous myocardial infarction. This study indicates that persons carrying a copy of the APOE4 allele are especially sensitive to cholesterol synthesis altering interventions. HMGCR gene expression in PBMCs has been shown to closely parallel liver gene expression [35]. If the higher expression of this gene and other SREBP-2-regulated cholesterol biosynthesis genes is present in liver as well, it may possibly explain why APOE4 carriers are especially sensitive to cholesterol synthesis altering interventions with respect to CVD risk. In this study, we describe changes in two pathways that may potentially contribute to the increased CVD risk that is observed in carriers of APOE4: (1) increased expression of IFN signaling and IFN target genes, and (2) increased expression of cholesterol biosynthesis genes. Several studies have examined a possible link between inflammation and cholesterol synthesis. Chen et al. [36] found that inflammatory stress causes intracellular cholesterol accumulation, and increases HMGCR gene and protein expression via the SREBP-2 pathway in mice both in vitro as well as in vivo. Furthermore, Zhao et al. [37] induced inflammatory stress in mice and in a human cell line and found increased cholesterol synthesis and SREBP2 and HMGCR gene and protein expression. Taking these findings and our gene expression results together, it might be possible that increased inflammatory stress, as indicated by 
increased IFN signaling in APOE4 carriers may have affected cholesterol biosynthesis gene expression. Further research is required, however, to explore the role of inflammatory stress and cholesterol biosynthesis as potential mechanisms for the increased CVD risk in APOE4 carriers as well as the relationship between these processes.

Although the expression of genes in the cholesterol biosynthesis pathway was significantly higher in APOE4 carriers compared to non-carriers, we did not observe a significant response to fish-oil supplementation in either group. When comparing carriers and non-carriers after supplementation the cholesterol biosynthesis pathway is no longer significantly differently expressed, suggesting that fish-oil might have affected gene expression of these genes to some extent. Possibly, these changes may have gone undetected due to the relatively low number of participants, especially in the APOE4 group.

A limitation of this study is that, due to the fact that it was not originally designed to answer the current research question, the groups are relatively small and not of the same size. We performed power calculations, which showed that we had sufficient power for the microarray analyses. Distinct differences in gene expression between carriers and non-carriers of the APOE4 allele, both at baseline as well as in the response to a 6-month fish-oil intervention were observed. Furthermore, several of the differences at baseline were confirmed in a larger group using qPCR. For our microarray analyses, we used a relatively high P-value cut-off of 0.05. Using FDR q-values for selecting the differentially expressed genes did not yield results in several comparisons. For this reason, we focused our analyses mainly on the pathway level. In this study, we opted not to include to placebo group in the analysis [15]. Firstly, because our study is focused on the differences between APOE4 carriers and non-carriers in the response to fish-oil. Secondly, adding this group, would further complicate this already quite complicated analysis and, as a consequence, reduce the readability of the paper. 
The opposite effect of fish-oil supplementation in APOE4 carriers and non-carriers that we found, can partially explain the heterogeneity that is seen in the responses to fish-oil supplementation on gene expression profiles and illustrates the importance of research on diet-gene interactions and research into personalized nutrition in general.

Our findings related to IFN signaling and cholesterol biosynthesis might explain part of the association between $A P O E 4$ and the increased risk of CVD that is observed in carriers of this allele. Additionally, with respect to our IFN signaling gene expression results we hypothesize that fish-oil supplementation may particularly benefit $A P O E 4$ carriers. 


\section{References}

[1] Mahley, R. W., Innerarity, T. L., Lipoprotein receptors and cholesterol homeostasis. Biochimica et biophysica acta 1983, 737, 197-222.

[2] Eisenberg, D. T., Kuzawa, C. W., Hayes, M. G., Worldwide allele frequencies of the human apolipoprotein E gene: climate, local adaptations, and evolutionary history. Am J Phys Anthropol 2010, 143, 100-111.

[3] Song, Y., Stampfer, M. J., Liu, S., Meta-analysis: apolipoprotein E genotypes and risk for coronary heart disease. Ann Intern Med 2004, 141, 137-147.

[4] Bennet, A. M., Di Angelantonio, E., Ye, Z., Wensley, F., et al., Association of apolipoprotein E genotypes with lipid levels and coronary risk. JAMA 2007, 298, 1300-1311.

[5] Khan, T. A., Shah, T., Prieto, D., Zhang, W., et al., Apolipoprotein E genotype, cardiovascular biomarkers and risk of stroke: systematic review and meta-analysis of 14,015 stroke cases and pooled analysis of primary biomarker data from up to 60,883 individuals. International journal of epidemiology 2013, 42, 475-492.

[6] Davignon, J., Gregg, R. E., Sing, C. F., Apolipoprotein E polymorphism and atherosclerosis. Arteriosclerosis 1988, 8, 1-21.

[7] Dallongeville, J., Lussier-Cacan, S., Davignon, J., Modulation of plasma triglyceride levels by apoE phenotype: a meta-analysis. J Lipid Res 1992, 33, 447-454.

[8] Perk, J., De Backer, G., Gohlke, H., Graham, I., et al., European Guidelines on cardiovascular disease prevention in clinical practice (version 2012). The Fifth Joint Task Force of the European Society of Cardiology and Other Societies on Cardiovascular Disease Prevention in Clinical Practice (constituted by representatives of nine societies and by invited experts). Eur Heart J 2012, 33, 1635-1701.

[9] Bucher, H. C., Hengstler, P., Schindler, C., Meier, G., N-3 polyunsaturated fatty acids in coronary heart disease: a meta-analysis of randomized controlled trials. Am J Med 2002, 112, 298-304.

[10] Rizos, E. C., Ntzani, E. E., Bika, E., Kostapanos, M. S., Elisaf, M. S., Association between omega-3 fatty acid supplementation and risk of major cardiovascular disease events: a systematic review and meta-analysis. JAMA 2012, 308, 1024-1033.

[11] Madden, J., Williams, C. M., Calder, P. C., Lietz, G., et al., The impact of common gene variants on the response of biomarkers of cardiovascular disease (CVD) risk to increased fish oil fatty acids intakes. Annu Rev Nutr 2011, 31, 203-234.

[12] Minihane, A. M., Khan, S., Leigh-Firbank, E. C., Talmud, P., et al., ApoE polymorphism and fish oil supplementation in subjects with an atherogenic lipoprotein phenotype.

Arterioscler Thromb Vasc Biol 2000, 20, 1990-1997.

[13] Olano-Martin, E., Anil, E., Caslake, M. J., Packard, C. J., et al., Contribution of apolipoprotein E genotype and docosahexaenoic acid to the LDL-cholesterol response to fish oil. Atherosclerosis 2010, 209, 104-110.

[14] Caslake, M. J., Miles, E. A., Kofler, B. M., Lietz, G., et al., Effect of sex and genotype on cardiovascular biomarker response to fish oils: the FINGEN Study. Am J Clin Nutr 2008, $88,618-629$.

[15] Bouwens, M., van de Rest, O., Dellschaft, N., Bromhaar, M. G., et al., Fish-oil supplementation induces antiinflammatory gene expression profiles in human blood mononuclear cells. Am J Clin Nutr 2009, 90, 415-424.

[16] de Mello, V. D., Kolehmanien, M., Schwab, U., Pulkkinen, L., Uusitupa, M., Gene expression of peripheral blood mononuclear cells as a tool in dietary intervention studies: What do we know so far? Mol Nutr Food Res 2012, 56, 1160-1172. 
[17] Jofre-Monseny, L., Loboda, A., Wagner, A. E., Huebbe, P., et al., Effects of apoE genotype on macrophage inflammation and heme oxygenase-1 expression. Biochemical and biophysical research communications 2007, 357, 319-324.

[18] Baitsch, D., Bock, H. H., Engel, T., Telgmann, R., et al., Apolipoprotein E induces antiinflammatory phenotype in macrophages. Arterioscler Thromb Vasc Biol 2011, 31, 11601168.

[19] Martinez-Morillo, E., Hansson, O., Atagi, Y., Bu, G., et al., Total apolipoprotein E levels and specific isoform composition in cerebrospinal fluid and plasma from Alzheimer's disease patients and controls. Acta neuropathologica 2014, 127, 633-643.

[20] van de Rest, O., Geleijnse, J. M., Kok, F. J., van Staveren, W. A., et al., Effect of fish oil on cognitive performance in older subjects: a randomized, controlled trial. Neurology 2008, $71,430-438$.

[21] Hixson, J. E., Vernier, D. T., Restriction isotyping of human apolipoprotein E by gene amplification and cleavage with HhaI. J Lipid Res 1990, 31, 545-548.

[22] Lin, K., Kools, H., de Groot, P. J., Gavai, A. K., et al., MADMAX - Management and analysis database for multiple omics experiments. Journal of integrative bioinformatics 2011, 8, 160.

[23] Irizarry, R. A., Bolstad, B. M., Collin, F., Cope, L. M., et al., Summaries of Affymetrix GeneChip probe level data. Nucleic Acids Res 2003, 31, e15.

[24] Gentleman, R., Carey, V., Huber, W., Irizarry, R., et al., Bioinformatics and

Computational Biology Solutions Using R and Bioconductor, Springer New York 2005, pp. 397-420.

[25] Subramanian, A., Tamayo, P., Mootha, V. K., Mukherjee, S., et al., Gene set enrichment analysis: a knowledge-based approach for interpreting genome-wide expression profiles. Proc Natl Acad Sci U S A 2005, 102, 15545-15550.

[26] Drabe, N., Zund, G., Grunenfelder, J., Sprenger, M., et al., Genetic predisposition in patients undergoing cardiopulmonary bypass surgery is associated with an increase of inflammatory cytokines. Eur J Cardiothorac Surg 2001, 20, 609-613.

[27] Tziakas, D. N., Chalikias, G. K., Antonoglou, C. O., Veletza, S., et al., Apolipoprotein E genotype and circulating interleukin-10 levels in patients with stable and unstable coronary artery disease. J Am Coll Cardiol 2006, 48, 2471-2481.

[28] McLaren, J. E., Ramji, D. P., Interferon gamma: a master regulator of atherosclerosis. Cytokine Growth Factor Rev 2009, 20, 125-135.

[29] Irons, R., Fritsche, K. L., Omega-3 polyunsaturated fatty acids impair in vivo interferongamma responsiveness via diminished receptor signaling. J Infect Dis 2005, 191, 481-486.

[30] Freund-Levi, Y., Hjorth, E., Lindberg, C., Cederholm, T., et al., Effects of omega-3 fatty acids on inflammatory markers in cerebrospinal fluid and plasma in Alzheimer's disease: the OmegAD study. Dement Geriatr Cogn Disord 2009, 27, 481-490.

[31] Minihane, A. M., Jofre-Monseny, L., Olano-Martin, E., Rimbach, G., ApoE genotype, cardiovascular risk and responsiveness to dietary fat manipulation. Proc Nutr Soc 2007, 66, 183-197.

[32] Goldstein, J. L., Brown, M. S., Regulation of the mevalonate pathway. Nature 1990, 343, 425-430.

[33] Horton, J. D., Goldstein, J. L., Brown, M. S., SREBPs: activators of the complete program of cholesterol and fatty acid synthesis in the liver. J Clin Invest 2002, 109, 1125 1131.

[34] Gerdes, L. U., Gerdes, C., Kervinen, K., Savolainen, M., et al., The apolipoprotein epsilon4 allele determines prognosis and the effect on prognosis of simvastatin in survivors of myocardial infarction : a substudy of the Scandinavian simvastatin survival study. Circulation 2000, 101, 1366-1371. 
[35] Powell, E. E., Kroon, P. A., Low density lipoprotein receptor and 3-hydroxy-3methylglutaryl coenzyme A reductase gene expression in human mononuclear leukocytes is regulated coordinately and parallels gene expression in human liver. J Clin Invest 1994, 93, 2168-2174.

[36] Chen, Y., Ku, H., Zhao, L., Wheeler, D. C., et al., Inflammatory stress induces statin resistance by disrupting 3-hydroxy-3-methylglutaryl-CoA reductase feedback regulation. Arterioscler Thromb Vasc Biol 2014, 34, 365-376.

[37] Zhao, L., Chen, Y., Tang, R., Chen, Y., et al., Inflammatory stress exacerbates hepatic cholesterol accumulation via increasing cholesterol uptake and de novo synthesis. Journal of gastroenterology and hepatology 2011, 26, 875-883.

\section{Author contributions}

The authors' contributions were as follows-LCPGMdG and JMG: designed the trial; OvdR: conducted the trial; JCM and LAA: designed the research; JCM: conducted the research, analyzed the data and wrote the manuscript; and LAA, MM and MR: critically reviewed the manuscript.

\section{Acknowledgements}

This work was supported by the Danish Council for Strategic Research [DSF 0603-004193]; Arla Foods Ingredients Group P/S; the Danish Dairy Research Foundation; the Dutch Dairy Association; and the Netherlands Organization for Health Research and Development [ZonMw, 6100.0004].

\section{Conflict of Interest}

The authors declare that there are no conflicts of interest. 


\section{Figures and tables}

Figure 1. Flow chart of gene selection methods and number of significantly differentially expressed genes. Genes were found to be significantly different if $\mathrm{P}<0.05$.

Figure 2. Top 10 differentially expressed pathways at baseline between APOE4 carriers and non-carriers. Top 10 was based on the lowest P-value. Total genes: total number of genes in the pathway, up (red): number of genes with significantly higher expression in APOE4 carriers compared to non-carriers, down (green): number of genes with significantly lower expression in APOE4 carriers compared to non-carriers.

Figure 3. Genes of which the expression is known to be regulated by IFNA2, IFNG and IFNL1 that are significantly differentially expressed at baseline in APOE4 carriers compared to non-carriers. Red indicates a significantly higher expression, green indicates a significantly lower expression $(\mathrm{P}<0.05)$. Orange arrow, gene is predicted to be activated by regulator; blue arrow, gene is predicted to be inhibited by regulator; yellow arrow, gene expression of downstream gene is inconsistent with predicted state.

Figure 4. Top 10 pathways differentially regulated by fish-oil supplementation in APOE4 carriers compared to non-carriers. The 10 canonical pathways with the lowest P-value are shown. The number of significantly up-regulated and down-regulated genes by fish-oil supplementation in APOE4 carriers and APOE4 non-carriers are shown. Total genes: total number of genes in the pathway, up (red): number of genes with significantly higher expression after intervention compared to before, down (green): number of genes with significantly lower expression after intervention compared to before. 
Figure 5. Heatmap depicting individual gene expression changes by fish-oil supplementation of genes that are significantly differently changed between carriers and non-carriers of APOE4 in response to fish-oil supplementation and are known to be regulated by IFNL1, IFNA2 and IFNG. Log-ratios of the expression after and before supplementation are shown.

Figure 6. QPCR results of a selection of genes showing the log ratio of expression in APOE4 carriers compared to non-carriers. Values are mean \pm SEM. $* \mathrm{p}<0.05$ in carriers compared to non-carriers.

HMGCR, 3-hydroxy-3-methylglutaryl-CoA reductase; HMGCS1, 3-hydroxy-3methylglutaryl-CoA synthase 1; IFITM1, IFN induced transmembrane protein 1; STAT1, signal transducer and activator of transcription 1; TAP1, transporter 1, ATP-binding cassette, sub-family B. 
Table 1. Primer sequences used to quantify gene expression by qPCR.

\begin{tabular}{lll}
\hline Gene & Forward primer & Reverse Primer \\
\hline HMGCR & TGATTGACCTTTCCAGAGCAAG & CTAAAATTGCCATTCCACGAGC \\
HMGCS1 & CAGAAGAACTTACGCTCGGC & TCTTGGCAGGGCTTGGAATA \\
IFITM1 & AGGGACAGGAAGATGGTTGG & AATCAGGGCCCAGATGTTCA \\
RPLP0 & CAGATTGGCTACCCAACTGTT & GGGAAGGTGTAATCCGTCTCC \\
STAT1 & GAGAGTCTGCAGCAAGTTCG & GGAAAAGACTGAAGGTGCGG \\
TAP1 & ATTTGAGTACCTGGACCGCA & GCCCCTGTAGCACTAAGACA \\
\hline
\end{tabular}


Table 2. Baseline characteristics of the 23 participants of the high fish-oil supplementation group in which PBMC gene expression microarrays were performed. ${ }^{\mathrm{a}}$

\begin{tabular}{|c|c|c|}
\hline & APOE4 carriers $(\mathrm{N}=8)$ & APOE4 non-carriers $(\mathrm{N}=15)$ \\
\hline Age & $69 \pm 3$ & $69 \pm 3$ \\
\hline$M / F$ & $5 / 3$ & $10 / 5$ \\
\hline$M / F \%$ & $62 / 38$ & $67 / 33$ \\
\hline Heigth $(\mathrm{cm})$ & $170 \pm 8$ & $173 \pm 6$ \\
\hline Weight (kg) & $77 \pm 7$ & $78 \pm 14$ \\
\hline $\mathrm{BMI}\left(\mathrm{kg} / \mathrm{m}^{2}\right)$ & $27.1 \pm 3.0$ & $26.1 \pm 3.4$ \\
\hline Waist circumference $(\mathrm{cm})$ & $96 \pm 6$ & $96 \pm 14$ \\
\hline Total cholesterol $(\mathrm{mmol} / \mathrm{l})$ & $6.0 \pm 0.6$ & $5.4 \pm 0.9$ \\
\hline Triglycerides (mmol/l) & $1.2 \pm 0.2$ & $0.9 \pm 0.4$ \\
\hline LDL-cholesterol (mmol/l) & $4.1 \pm 0.5$ & $3.5 \pm 0.8$ \\
\hline HDL-cholesterol $(\mathrm{mmol} / \mathrm{l})$ & $1.5 \pm 0.4$ & $1.5 \pm 0.3$ \\
\hline $\mathrm{hsCRP}(\mathrm{mg} / \mathrm{L})^{\mathrm{b}}$ & $2.0 \pm 1.9$ & $3.3 \pm 3.2$ \\
\hline
\end{tabular}

${ }^{\mathrm{a}}$ Data presented as mean $\pm \mathrm{sd}$. Baseline values were not significantly different between carriers and non-carriers at $\mathrm{P}<0.05$.

${ }^{\mathrm{b}}$ hsCRP, high sensitivity C-reactive protein. 
Table 3. Baseline comparison of APOE4 carriers and non-carriers of genes involved in cholesterol biosynthesis, IFN signaling and IFN targets. ${ }^{\text {a }}$

\begin{tabular}{|c|c|c|c|c|}
\hline Gene & $\begin{array}{c}\text { Entrez } \\
\text { ID }\end{array}$ & Description & P-value & Fold change \\
\hline \multicolumn{5}{|l|}{ Cholesterol synthesis } \\
\hline DHCR24 & 1718 & 24-dehydrocholesterol reductase & 0.02 & 1.24 \\
\hline GGPS1 & 9453 & $\begin{array}{l}\text { geranylgeranyl diphosphate synthase } 1 \\
\text { hydroxyacyl-CoA dehydrogenase/3-ketoacyl-CoA thiolase/enoyl-CoA hydratase }\end{array}$ & 0.05 & 1.21 \\
\hline HADHA & 3030 & $\begin{array}{l}\text { (trifunctional protein), alpha subunit } \\
\text { hydroxyacyl-CoA dehydrogenase/3-ketoacyl-CoA thiolase/enoyl-CoA hydratase }\end{array}$ & $<0.01$ & -1.12 \\
\hline HADHB & 3032 & (trifunctional protein), beta subunit & 0.04 & -1.08 \\
\hline HMGCR & 3156 & 3-hydroxy-3-methylglutaryl-CoA reductase & 0.02 & 1.24 \\
\hline HMGCS1 & 3157 & 3-hydroxy-3-methylglutaryl-CoA synthase 1 (soluble) & $<0.01$ & 1.18 \\
\hline IDI1 & 3422 & isopentenyl-diphosphate delta isomerase 1 & $<0.01$ & 1.14 \\
\hline \multicolumn{5}{|l|}{ IFN signaling } \\
\hline IFNAR1 & 3454 & interferon (alpha, beta and omega) receptor 1 & $<0.01$ & 1.26 \\
\hline IRF9 & 10379 & interferon regulatory factor 9 & $<0.01$ & 1.26 \\
\hline MX1 & 4599 & MX dynamin-like GTPase 1 & 0.02 & 1.73 \\
\hline PSMB8 & 5696 & proteasome (prosome, macropain) subunit, beta type, 8 & $<0.01$ & 1.13 \\
\hline \multicolumn{5}{|l|}{ IFN targets } \\
\hline IFI35 & 3430 & interferon-induced protein 35 & 0.01 & 1.33 \\
\hline IFIT1 & 3434 & interferon-induced protein with tetratricopeptide repeats 1 & $<0.01$ & 2.54 \\
\hline IFIT3 & 3437 & interferon-induced protein with tetratricopeptide repeats 3 & 0.01 & 2.01 \\
\hline IFITM1 & 8519 & interferon induced transmembrane protein 1 & $<0.01$ & 1.17 \\
\hline IFNAR1 & 3454 & interferon (alpha, beta and omega) receptor 1 & $<0.01$ & 1.26 \\
\hline IRF9 & 10379 & interferon regulatory factor 9 & $<0.01$ & 1.26 \\
\hline MX1 & 4599 & MX dynamin-like GTPase 1 & 0.02 & 1.73 \\
\hline
\end{tabular}

${ }^{a}$ Genes selection is based on Ingenuity canonical pathways.

${ }^{\mathrm{b}} \mathrm{P}$-values and fold changes for the comparison between APOE4 carriers and non-carriers are shown. P-values were considered significant when $\mathrm{P}<0.05$. 
Table 4. Top 10 potential upstream regulators $^{\mathrm{a}}$ explaining the baseline differences in gene expression between $A P O E 4$ carriers and non-carriers. ${ }^{\mathrm{b}}$

\begin{tabular}{llllll} 
Upstream Regulator & Fold Change & Molecule Type & Predicted Activation State & Activation z-score & P-value of overlap \\
\hline PRL & -1.17 & cytokine & Activated & 6.76 & $3.77 \mathrm{E}-33$ \\
IFNL1 & 1.02 & cytokine & Activated & 6.21 & $2.14 \mathrm{E}-29$ \\
IFNA2 & -1.03 & cytokine & Activated & 6.17 & $3.06 \mathrm{E}-23$ \\
MAPK1 & -1.02 & kinase & Inhibited & -6.13 & $2.13 \mathrm{E}-18$ \\
CNOT7 & 1.03 & transcription regulator & Inhibited & -2.43 & $1.31 \mathrm{E}-15$ \\
IL1RN & 1.13 & cytokine & Inhibited & -4.64 & $1.89 \mathrm{E}-15$ \\
IFNG & 1.32 & cytokine & Activated & 5.45 & $5.33 \mathrm{E}-12$ \\
EIF2AK2 & 1.49 & kinase & Activated & 3.75 & $1.92 \mathrm{E}-09$ \\
Interferon alpha & & group & Activated & 4.01 & $2.90 \mathrm{E}-08$ \\
IFNB1 & & cytokine & Activated & 3.44 & $1.86 \mathrm{E}-06$ \\
\hline
\end{tabular}

${ }^{\text {a }}$ Ingenuity upstream regulator analysis identifies transcriptional regulators that can potentially explain the observed changes in gene expression.

${ }^{\mathrm{b}}$ The 10 regulators with the lowest $\mathrm{P}$-value and a significant $\mathrm{Z}$ score are shown. Fold change for the upstream regulator is shown if significantly differentially expressed. $\mathrm{Z}$ scores predict the activation state of the regulator and are based on the gene expression of its downstream genes. Upstream regulators with z scores $>2$ are considered activated and regulators with $\mathrm{z}$ scores $<2$ are considered inhibited. P-values of overlap are calculated using Fisher's exact test. 
Table 5. Top 10 potential upstream regulators ${ }^{\mathrm{a}}$ identified for the difference in gene expression changes in response to 6 months of fish-oil supplementation between carriers and non-carriers of the APOE4 SNP. ${ }^{\mathrm{b}}$

\begin{tabular}{|c|c|c|c|c|c|c|c|c|}
\hline \multirow[b]{2}{*}{ Regulator } & \multirow[b]{2}{*}{ Molecule Type } & \multirow{2}{*}{$\begin{array}{l}\text { Difference in } \\
\text { response to fish-oil } \\
\text { P-value }\end{array}$} & \multicolumn{3}{|c|}{ Response in $A P O E 4$ carriers } & \multicolumn{3}{|c|}{ Response in $A P O E 4$ non-carriers } \\
\hline & & & $\begin{array}{c}\text { Activation } \\
\text { state }\end{array}$ & $\mathrm{Z}$ score & P-value & Activation state & $\mathrm{Z}$ score & P-value \\
\hline PRL & cytokine & 4.09E-39 & Inhibited & -4.59 & $2.90 \mathrm{E}-20$ & & 1.92 & $1.89 \mathrm{E}-03$ \\
\hline IFNL1 & cytokine & $2.31 \mathrm{E}-30$ & Inhibited & -4.53 & $5.84 \mathrm{E}-11$ & Activated & 3.50 & $1.18 \mathrm{E}-03$ \\
\hline IFNA2 & cytokine & $2.98 \mathrm{E}-28$ & Inhibited & -5.01 & $1.67 \mathrm{E}-15$ & Activated & 2.36 & $1.71 \mathrm{E}-04$ \\
\hline MAPK1 & kinase & $2.24 \mathrm{E}-23$ & Activated & 5.77 & $3.44 \mathrm{E}-15$ & Inhibited & -3.93 & $7.55 \mathrm{E}-03$ \\
\hline CNOT7 & transcription regulator & $2.87 \mathrm{E}-19$ & Activated & 2.22 & 2.13E-08 & & & Not significant \\
\hline IL1RN & cytokine & 4.34E-19 & Activated & 3.30 & 3.31E-08 & Inhibited & -2.52 & 4.69E-03 \\
\hline IFNG & cytokine & $2.80 \mathrm{E}-17$ & Inhibited & -3.97 & $7.67 \mathrm{E}-13$ & Inhibited & -2.52 & 4.69E-03 \\
\hline TGM2 & enzyme & $3.11 \mathrm{E}-13$ & Inhibited & -4.65 & $1.18 \mathrm{E}-13$ & Inhibited & -2.12 & $1.05 \mathrm{E}-06$ \\
\hline EIF2AK2 & kinase & $3.30 \mathrm{E}-13$ & Inhibited & -3.71 & 8.84E-06 & & 0.31 & $2.72 \mathrm{E}-02$ \\
\hline STAT2 & transcription regulator & $2.59 \mathrm{E}-12$ & & & $1.47 \mathrm{E}-05$ & & & $2.17 \mathrm{E}-02$ \\
\hline
\end{tabular}

${ }^{a}$ Ingenuity upstream regulator analysis identifies transcriptional regulators that can potentially explain the observed changes in gene expression.

${ }^{\mathrm{b}}$ The 10 regulators with the lowest $\mathrm{P}$-value and a significant $\mathrm{Z}$ score are shown for the difference in gene expression changes in response to 6 months of fish-oil supplementation between $A P O E 4$ carriers and non-carriers are shown. $\mathrm{Z}$ scores, activation state and $\mathrm{p}$-values are depicted for the responses within the groups of APOE4 carriers and the non-carriers. $\mathrm{Z}$ scores predict the activation state of the regulator and are based on the gene expression of its downstream genes. Upstream regulators with z scores $>2$ are considered activated and regulators with z scores $<2$ are considered inhibited. P-values of overlap are calculated using Fisher's exact test. 